\title{
Paper 0-5
}

\section{Power deposition on the DIII-D inner wall limiter}

\author{
P.C. Stangeby ${ }^{\mathrm{a}, *}$, C.K. Tsui ${ }^{\mathrm{a}}$, C.J. Lasnier ${ }^{\mathrm{b}}$, J.A. Boedo ${ }^{\mathrm{c}}$, J.D. Elder ${ }^{\mathrm{a}}$, M. Kocan ${ }^{\mathrm{d}}$,
} A.W. Leonard ${ }^{\mathrm{e}}$, A.G. McLean ${ }^{\mathrm{b}}$, R.A. Pitts ${ }^{\mathrm{d}}$, and D.L. Rudakov ${ }^{\mathrm{c}}$

\author{
${ }^{a}$ University of Toronto Institute for Aerospace Studies, Toronto, M3H 5T6, Canada \\ ${ }^{b}$ Lawrence Livermore National Laboratory, 7000 East Avenue, Livermore, CA, 94550, USA \\ ${ }^{c}$ University of California San Diego, 9500 Gilman Drive, La Jolla, CA, 92093-0417, USA \\ ${ }^{d}$ ITER Organization, Route de Vinon sur Verdon - 13115 St Paul Lez Durance - France \\ ${ }^{e}$ General Atomics, P.O. Box 85608, San Diego, CA, 92186-5608, USA
}

\begin{abstract}
ITER will use the shaped inner column as startup/rampdown limiter. An exploratory experiment was performed in DIII-D using a similarly shaped inner wall limiter, looking for evidence of a short fall-off feature, $\lambda^{\text {short }}$, near the last closed flux surface. Measurements were made on the high field side (HFS) using infrared thermography and a Langmuir Swing-Probe. In some cases clear evidence was found for a narrow feature $\lambda^{\text {short }} \sim \rho_{\text {pol }}^{\mathrm{D}^{+}} \sim$ a few mm, and of strength_The ratio $q \| q_{\|}^{\text {short }} / q_{\| 0}^{\text {long }}$ was mostly between 0.1 and $0.6 .0 .5 \pm$ a factor of 5 .
\end{abstract} Measurements made on the low field side and bottom side showed little clear indication of any narrow feature.

Abstract length (150 words): 104 currently

PACS: $52.40 . \mathrm{Hf}, 52.55 . \mathrm{Fa}, 52.55 . \mathrm{Rk}$

PSI-21 keywords: cross-field transport, DIII-D, limiter, probes, power deposition, thermography

*Corresponding and presenting author address: c/o General Atomics, PO Box 85608, San Diego, California 92186-5608, USA

*Corresponding and presenting author e-mail: stangeby@fusion.gat.com 


\section{Introduction}

Power deposition, $\mathrm{q}_{\text {surf }}$, on the inner wall limiter (IWL) of DIII-D, was measured by infrared (IR) thermography and calculated from plasma profiles measured by probes and Thomson scattering for 6 ohmic discharges, Table 1. The objective of the experiment was to check the assumptions made in defining the shape of the ITER IWL, in particular to see if the radial gradient of the parallel power flux density, $\mathrm{q}_{\|}$, increases near the last closed flux surface (LCFS) as observed in other tokamaks [1-4], also occurs in DIII-D, which has an IWL resembling closely the ITER IWL.

Although it is generally thought that $\lambda_{\mathrm{sOL}}$ is set by plasma turbulence, some neoclassical theories predict $\lambda_{\mathrm{SOL}} \approx \underset{\mathrm{D}^{+}}{\mathrm{pol}}[5-10] \sim$ a few mm. Such a $\lambda_{\mathrm{SOL}}^{\text {short }}$ component could be difficult to observe on the low field side (LFS) due to the high turbulence there, but might manifest itself on the HFS, which was observed to be rather quiescent (e.g. low noise swing-probe IV characteristics). Measurements were made using an IR camera system viewing the DIII-D IWL and using (i) a new Langmuir swing-probe (SP) on the HFS, (ii) a reciprocating Langmuir probe (RCP) on the LFS, (iii) an RCP entering from the bottom of the poloidal cross section, (iv) a Thomson scattering (TS) system on the LFS, and (v) a lower TS ["Divertor Thomson" (DTS)] system (Fig. 1). The latter diagnostics measured radial profiles of $n_{e}$ and $T_{e}$ from which $\mathrm{q}_{\|}$can be calculated assuming, e.g. $\mathrm{T}_{\mathrm{e}}=\mathrm{T}_{\mathrm{i}}$ and sheath heat transmission coefficient $\gamma_{\text {sheath }}=7$. If a $\mathrm{q}_{\|}^{\text {short }}$ component exists and is due to neoclassical ion transport, then a narrow component should also exist for parallel ion particle flux density, $\Gamma_{\|}^{\text {short }}$. The most basic and reliable measurement made by Langmuir probes is the ion saturation current flux density, $\mathrm{J}_{\text {sat }}=\mathrm{e} \Gamma_{\|}$. In contrast, extraction of a value of $\mathrm{q}_{\|}$from probe measurements requires: (i) using the probe-measured $\mathrm{T}_{\mathrm{e}}$, which is often noisier than the $\mathrm{J}_{\text {sat }}$; (ii) making assumptions about the often 
unmeasured $T_{i}$ and thus of the value of $\gamma_{\text {sheath. }}$. Therefore we start with SP evidence for a $\mathrm{J}_{\text {sat }}^{\text {short }}$ component.

On the L(low)FS or M(mid)FS there was little clear indication of any narrow feature; however, the plasma positions used were not optimal for accessing the LFS/MFS diagnostics and further experiments are required for definitive conclusions. These data are not discussed further here.

\section{Swing-probe measurements near the IWL of the radial profiles of parallel particle}

\section{and power flux densities}

We illustrate with shot 155646 at $2 \mathrm{~s}$ for the lower arc (the inward and upper swing) of the SP as representative, Fig. 2. The best double exponential fit, $J_{\text {sat }}(x)=J_{\text {sat } 0}^{\text {short }} \mathrm{e}^{-\mathrm{x} / \lambda_{\mathrm{J}}^{\text {short }}}+\mathrm{J}_{\text {sat } 0}^{\text {long }} \mathrm{e}^{-\mathrm{x} / \lambda_{\mathrm{J}}^{\text {long }}}$, had $\lambda_{\mathrm{J}}^{\text {long }}=50 \mathrm{~mm}, \lambda_{\mathrm{J}}^{\text {short }}=3.8 \mathrm{~mm}, J_{\text {sat0 }}^{\text {long }}=23.7 \mathrm{kA} / \mathrm{m}^{2}, \underset{-}{*} \mathrm{~J}_{\text {sat0 }}^{\text {short }}=10.7 \mathrm{kA} / \mathrm{m}^{2} \stackrel{*}{*}$, thus $\mathrm{R}_{\mathrm{J}_{\mathrm{sat}}} \equiv \mathrm{J}_{\text {sat0 }}^{\text {short }} / \mathrm{J}_{\text {sat0 }}^{\text {long }}=0.45$. [ $\mathrm{J}_{\text {sat0 }}$ values are at the IMP (inner midplane); the $\lambda_{\mathrm{J}}$ values are mapped to the OMP (outer MP); this convention is used generally in this paper.] The values of $\mathrm{R}_{\mathrm{J}_{\text {sat }}}$ for each of the shots were found to be close to the value for $\mathrm{R}_{\mathrm{q}_{\|}}$given in Table 2 .

Figure 3(a) shows the radial profile of $T_{e}$ measured by the SP for 155646 at $2 \mathrm{~s}$ and Fig. 3(b) the parallel power flux density, $\mathrm{q}_{\|}$, calculated using individual pairs of $\left(\mathrm{T}_{\mathrm{e}}, \mathrm{J}_{\mathrm{sat}}\right)$, and assuming $\gamma_{\text {sheath }}=7$, i.e. $\mathrm{q}_{\|}=7 \mathrm{~T}_{\mathrm{e}} \mathrm{J}_{\text {sat }}$. It was found for all the $\mathrm{q}_{\|}$profiles calculated in this way that there is no clear-cut prima facie evidence for the existence of a double exponential shape, i.e. nothing very obvious jumps off the page. For the most part, the data seem to be reasonably well approximated by single exponentials. Nevertheless there is a suggestion in Fig. 3(b) of a $\lambda_{\mathrm{q}}^{\text {short }}$ component near the LCFS, warranting a more careful look.

A drawback of the foregoing method of calculating $\mathrm{q}_{\|}$is that the $\mathrm{T}_{\mathrm{e}}$ values have larger scatter than the $J_{\text {sat }}$ values. Thus when $q_{\|}$is calculated using the specific $T_{e}$ value that 
corresponds to the specific $\mathrm{J}_{\text {sat }}$ value for individual probe $\mathrm{I}-\mathrm{V}$ characteristics, then the $\mathrm{q}_{\|}$values are also scattered, making it difficult to detect any $\lambda_{\mathrm{q}}^{\text {short }}$ component. As seen in Fig. 2, often there is clear structure in the $\mathrm{J}_{\text {sat }}$ profile, while the $T_{\mathrm{e}}$ profiles, although more scattered, seem relatively featureless, e.g. Fig. 3(a). Therefore, a second method was used to calculate $q_{\|}$: first, for each shot a best single-exponential fit was made to the $T_{e}$ profile, Fig. 3(a), and then values from that best-fit were used to calculate $\mathrm{q}_{\|}$, Fig. 3(c), where the data have been bin-averaged for greater clarity.

The single-exponential best fit to the $T_{e}$ data in Fig. 3(a) gives $T_{e 0}=31 \mathrm{eV}$ and $\lambda_{\mathrm{T}_{\mathrm{e}}}=40 \mathrm{~mm}$. The $1 \mathrm{~mm}$ bin-averaged $\mathrm{q}_{\|}$values in Fig. 3(c) were best fit to a double exponential (solid line), giving $\lambda_{\mathrm{q}}^{\text {long }}=23 \mathrm{~mm}, \quad \lambda_{\mathrm{q}}^{\text {short }}=4.2 \mathrm{~mm}, \quad \mathrm{q}_{|| 0}^{\text {long }}=5.12 \mathrm{MW} / \mathrm{m}^{2}, \quad q_{\| 0}^{\text {short }}=2.93 \mathrm{MW} / \mathrm{m}^{2}$ thus $\mathrm{R}_{\mathrm{q}_{\|}} \equiv \mathrm{q}_{\| 0}^{\text {short }} / \mathrm{q}_{\| 0}^{\text {long }}=0.57$. Results for all the shots are given in Table 2 which also includes values of $\rho_{\mathrm{pol}}^{\mathrm{D}^{+}}$calculated for $B_{\mathrm{pol}}^{\mathrm{IMP}}$ and $\mathrm{T}_{\mathrm{i}} / \mathrm{T}_{\mathrm{e} 0}=4$, a typical value for limiter SOLS [11]. As can be seen $\lambda_{\mathrm{q}}^{\text {short }} / \rho_{\mathrm{pol}}^{\mathrm{D}^{+}} \sim 1$, possibly indicating that the short feature is due to a neo-classical effect; direct measurements of $\mathrm{T}_{\mathrm{i}}$ will be required for more definitive conclusions.

\section{Radial profiles of $q_{\|}$from IR thermographic measurements of the $2 D$ spatial distribution of deposited power on the IWL}

The DIII-D inner wall is clad with $\mathrm{n}=48$ columns of flat, $14 \mathrm{~cm}$ square graphite tiles. Thus the IWL is comprised of 48 individual roof-top shape limiters of roof apex angle $\alpha_{\text {apex }}=360^{\circ} / 48=7.5^{\circ}$. The DIII-D limiter geometry is therefore similar to ITER's, which is also an approximation to a continuous toroidal limiter, involving $n=36$ individual limiters in ITER's case. The angle of incidence (between the parallel direction and the limiter surface) at the apex is similar: in DIII-D $\alpha_{\mathrm{inc}}^{\mathrm{apex}}=7.5^{\circ} / 2=3.75^{\circ}$; in ITER $\alpha_{\mathrm{inc}}^{\mathrm{apex}} \sim 5^{\circ}$. In DIII-D (resp. ITER) the apexes 
are at $\mathrm{R} \sim 1$ (resp. 4) $\mathrm{m}$ which defines $\mathrm{R}_{\mathrm{IWL}}$. The depth of the modulation of the IWL is greater in ITER, $\sim 30 \mathrm{~mm}$, than in DIII-D, 2 mm. For shot 155648 at $3.5 \mathrm{~s}$ where the plasma contact with the IWL occurred at $\mathrm{Z}=-0.25 \mathrm{~m}$, Fig. 1, 2D contour plots of $\alpha_{\text {inc }}$ and $\delta$ are given in Fig. 4; $\alpha_{\text {inc }}=$ angle between the limiter surface and $\vec{B}$ which is upward and to the left here; $\Delta \mathrm{r}_{\mathrm{sep}}=$ radial distance from the limiter surface to the LCFS, mapped to OMP.

The deposited power flux density, $\mathrm{q}_{\text {surf }}$ was obtained from infrared thermography (Fig. 5). As can be seen, there is no evidence of shadowing; the observed pattern is similar to that calculated for no shadowing (Fig. 4 right). The plasma wetting of the tile surfaces which should be shadowed according to standard line-tracing is hypothesized to be due to the very shallow limiter modulation, which is only $\sim 1 \mathrm{~mm}$ on average, thus comparable to the thickness of the magnetic pre-sheath. While this is advantageous for power-handling (since the wetted area is doubled), it is a complication here and we therefore use data only from the regions which are not shadowed according to line-tracing.

We focus the IR analysis here on the lower-right quadrants of the two peak regions, analyzing vertical slices of $\mathrm{q}_{\text {surf }}$ data at 6 toroidal locations and for $\mathrm{Z}<\mathrm{Z}_{\mathrm{c}}$. The values of $\mathrm{q}_{\|}$are calculated using $\mathrm{q}_{\|}=\mathrm{q}_{\text {dep }} / \sin \alpha_{\text {inc }}$. Results are shown in Fig. 6 . The six $\mathrm{q}_{\| \text {ir }}$ profiles in Fig. 6 were averaged, Fig. 7, which also shows the $\mathrm{q}_{\|}$profiles from the two arcs of the SP. Since the interest here is on the shape rather than magnitude of the $\mathrm{q}_{\|}$profiles, each of the $\mathrm{q}_{\|}$profiles in Fig. 7 were normalized to their values at the LCFS. The best-fit double-exponential to the $q_{\| \text {ir }}$ profile gave $\lambda_{\mathrm{q}}^{\text {long }}=18 \mathrm{~mm}, \lambda_{\mathrm{q}}^{\text {short }}=2.3 \mathrm{~mm}$ and $\mathrm{R}_{\mathrm{q}_{\|}}=2.07$ which agrees well with the average values for the two arcs of the SP: $\lambda_{\mathrm{q}}^{\text {long }}=18 \mathrm{~mm}, \lambda_{\mathrm{q}}^{\text {short }}=2.2 \mathrm{~mm}$ and $\mathrm{R}_{\mathrm{q}_{\|}}=1.69$. 


\section{Summary}

In some cases Swing-Probe and IR measurements made on the HFS of DIII-D IWL discharges provide clear evidence for a narrow feature $\lambda_{q, \Gamma}^{\text {short }} \sim$ a few $\mathrm{mm}$, and of strength $q_{\| 0}^{\text {short }} / q_{\| 0}^{\text {long }} \sim \Gamma_{\| 0}^{\text {short }} / \Gamma_{\| 0}^{\text {long }} \sim 0.5 \pm$ a factor of $5 . \lambda_{\mathrm{q}}^{\text {short }} / \rho_{\text {pol }}^{\mathrm{D}^{+}} \sim 1$, possibly indicating that the short feature is due to a neoclassical effect.

\section{Acknowledgment}

This work was supported in part by the US Department of Energy under DE-AC52-07NA27344, DE-FG02-07ER54917, and DE-FC02-04ER54698. DIII-D data shown in this paper can be obtained in digital format by following the links at https://fusion.gat.com/global/D3D_DMP. 


\section{References}

1. G. Arnoux et al., Nucl. Fusion 53 (2013) 073016

2. J. Horacek, this Conf. O4

3. F. Nespoli, this Conf P1-095

4. B. LaBombard, private communication

5. V.G. Petrov, Nucl. Fusion 24 (1984) 259.

6. A.V. Nedospasov, et al., Nucl. Fusion 25 (1985) 21.

7. F.L. Hinton and M.S. Chu, Nucl. Fusion 25 (1985) 345.

8. A.V. Chankin and V.A. Vershkov, J. Nucl. Mater. 162-164 (1989) 208.

9. M.A. Kovanen et al., Nucl Fusion 32 (1992) 863.

10. R. Goldston, Nucl. Fusion 52 (2012) 013009.

11. M. Kocan, et al., J. Nucl. Mater. 415 (2011) S1133. 
Table 1. Some properties of the DIII-D IWL discharges for times when the Swing-Probe was used.

\begin{tabular}{|c|l|l|c|c|c|c|c|c|}
\hline Shot & $\begin{array}{c}\text { Time } \\
(\mathrm{s})\end{array}$ & $\begin{array}{c}\mathrm{I}_{\mathrm{p}} \\
(\mathrm{MA})\end{array}$ & $\begin{array}{c}\mathrm{n}_{\mathrm{e}}(0) \\
\left(10^{19} / \mathrm{m}^{3}\right)\end{array}$ & $\kappa$ & $\begin{array}{c}\text { Top Gap } \\
(\mathrm{cm})\end{array}$ & $\begin{array}{c}\text { Bottom } \\
\mathrm{Gap} \\
(\mathrm{cm})\end{array}$ & $\begin{array}{c}\mathrm{P}_{\text {tot }} \\
(\mathrm{MW})\end{array}$ & $\begin{array}{c}\mathrm{P}_{\text {tot }^{-}} \\
(\mathrm{MW})\end{array}$ \\
\hline 155645 & 2 & 1.19 & 3.64 & 1.58 & 3 & 3 & 1.06 & 0.55 \\
\hline 155646 & 2 & 1.2 & 3.64 & 1.59 & 3 & 3 & 1.05 & 0.65 \\
\hline 155648 & 3.5 & 1.19 & 3.36 & 1.26 & $\mathrm{v}$ large & 6 & 1.06 & 0.74 \\
\hline 155649 & 3.5 & 0.61 & 1.79 & 1.24 & $\mathrm{v}$ large & 6 & 0.37 & 0.25 \\
\hline 155650 & 2 & 0.61 & 1.93 & 1.6 & 3 & 2.5 & 0.39 & 0.19 \\
\hline 155651 & 2 & 0.61 & 1.93 & 1.66 & 1.5 & 2.2 & 0.37 & 0.14 \\
\hline
\end{tabular}


Table 2. Parameters for best single-exponential fits to $\mathrm{T}_{\mathrm{e}}$ profiles measured by the Swing-Probe and for best double-exponential fits to $\mathrm{q}_{\|}$profiles calculated using individual values of $\mathbf{J}_{\text {sat }}$ and values from the fit to $\mathrm{T}_{\mathrm{e}} \cdot \rho_{\mathrm{pol}}^{\mathrm{D}^{+}}$calculated for $B_{\mathrm{pol}}^{\mathrm{IMP}}$ and $\mathrm{T}_{\mathrm{i}} / \mathrm{T}_{\mathrm{e} 0}=4$, a typical value for limiter SOLS [11].

\begin{tabular}{|c|c|c|c|c|c|c|c|c|c|c|c|c|}
\hline Shot & $\begin{array}{c}\mathrm{I}_{\mathrm{p}} \\
(\mathrm{MA})\end{array}$ & $\kappa$ & Arc & $\begin{array}{c}\mathrm{q}_{\| 0}^{\text {long }} \\
\left(\mathrm{MW} / \mathrm{m}^{2}\right)\end{array}$ & $\begin{array}{c}\mathrm{q}_{\| 0}^{\text {short }} \\
\left(\mathrm{MW} / \mathrm{m}^{2}\right)\end{array}$ & $\mathrm{R}_{\mathrm{q} \|}$ & $\begin{array}{c}\lambda_{\mathrm{q}}^{\text {long }} \\
(\mathrm{mm})\end{array}$ & $\begin{array}{c}\lambda_{\mathrm{q}}^{\text {short }} \\
(\mathrm{mm})\end{array}$ & $\begin{array}{c}T_{\mathrm{e} 0} \\
(\mathrm{eV})\end{array}$ & $\begin{array}{c}\lambda_{T_{\mathrm{e}}} \\
(\mathrm{mm})\end{array}$ & $\begin{array}{c}\rho_{\mathrm{pol}}^{+} \\
(\mathrm{mm})\end{array}$ & $\lambda_{\mathrm{q}}^{\text {short }} / \rho_{\mathrm{pol}}^{\mathrm{D}^{+}}$ \\
\hline 155645 & 1.2 & 1.60 & Lower & 4.23 & 0.46 & 0.11 & 28 & 2.6 & 27 & 50 & 3.0 & 0.88 \\
\hline 155645 & 1.2 & 1.60 & Upper & 3.42 & 1.81 & 0.53 & 18 & 3.6 & 23 & 33 & 2.7 & 1.32 \\
\hline 155646 & 1.2 & 1.60 & Lower & 5.12 & 2.93 & 0.57 & 23 & 4.2 & 31 & 40 & 3.2 & 1.33 \\
\hline 155646 & 1.2 & 1.60 & Upper & 4.20 & 0.77 & 0.18 & 14 & 1.5 & 25 & 23 & 2.9 & 0.53 \\
\hline 155648 & 1.2 & 1.25 & Lower & 6.10 & 3.82 & 0.63 & 21 & 2.3 & 37 & 44 & 3.9 & 0.60 \\
\hline 155648 & 1.2 & 1.25 & Upper & 3.63 & 9.93 & 2.74 & 15 & 2.1 & 32 & 25 & 3.6 & 0.59 \\
\hline 155649 & 0.6 & 1.25 & Lower & 1.85 & 1.32 & 0.71 & 30 & 2.4 & 29 & 55 & 6.9 & 0.88 \\
\hline 155649 & 0.6 & 1.25 & Upper & 1.82 & 0.34 & 0.19 & 22 & 1.2 & 25 & 57 & 6.4 & 0.19 \\
\hline 155650 & 0.6 & 1.60 & Lower & 2.26 & 0.74 & 0.33 & 18 & 3.8 & 31 & 29 & 6.3 & 0.61 \\
\hline 155650 & 0.6 & 1.60 & Upper & 1.74 & 0.97 & 0.56 & 17 & 3.3 & 26 & 23 & 5.8 & 0.57 \\
\hline 155651 & 0.6 & 1.66 & Lower & 1.18 & 0.068 & 0.058 & 21 & 6.7 & 18 & 38 & 5.7 & 1.17 \\
\hline 155651 & 0.6 & 1.66 & Upper & 0.95 & 0.10 & 0.11 & 19 & 4.0 & 14 & 43 & 4.8 & 0.67 \\
\hline Average & & & & & & $0.58 \pm 5 X$ & & & & & & $0.79 \pm 0.35$ \\
\hline
\end{tabular}


Fig. 1. Magnetic configuration for shot 155648 at $3.5 \mathrm{~s}$ showing the diagnostics used to measure the $\mathrm{q}_{\|}$profile.

Fig. 2. The radial profile of $\mathbf{J}_{\text {sat }}$ measured by the lower arc of the SP in Shot 155646 at 2 s: (a) unaveraged data, (b) $1 \mathrm{~mm}$ bin-average. The dashed lines are the best single-exponential fits to the data for $\mathrm{R}-\mathrm{R}_{\mathrm{sep}}>1 \mathrm{~cm}$, which provided the value for $\lambda_{\mathrm{J}_{\mathrm{sat}}}^{\text {long }}$ then used to obtain the best double-exponential fit (solid lines).

Fig. 3. (a) $\mathrm{T}_{e}$ measured by the lower arc of the swing-probe in Shot 155646 at $2 \mathrm{~s}$. (b) $\mathrm{q}_{\|}$, calculated using individual pairs of $\left(T_{e}, J_{\text {sat }}\right)$, (c) $q_{\|}$, calculated using individual values of $J_{\text {sat }}$ and values from the fit to $T_{e}$ from (a), then $1 \mathrm{~mm}$ bin-averaged. Solid lines in $(a, b)$ best fit single-exponentials. (c) the dashed line is the best single-exponential fit to the data for $R-R_{\text {sep }}$ $>1 \mathrm{~cm}$, which provided the value for $\lambda_{\mathrm{q}}^{\text {long }}$ then used to obtain the best double-exponential fit (solid curved line).

Fig. 4. Shot 155648 at $3.5 \mathrm{~s}$ when the LCFS-IWL contact occurred at $Z_{c}=-0.25 \mathrm{~m}$. (Left) $\alpha_{\text {inc }}$, (middle) $\Delta r_{\text {sep }}$, (right) calculated $q_{\text {surf }}$ for the parameters in Table 2; shadowing not included. Two columns of tiles are shown with roof apexes at toroidal distance $t=0$ and $\pm 0.14 \mathrm{~m}$. The maximum value of $\alpha_{\text {inc }} \sim 3.75^{\circ}$, occurs just to the sides of each limiter apex with $\alpha_{\text {inc }} \sim 0$ midway between apexes ( $\left|\alpha_{\text {inc }}\right|$ is plotted here). 
Fig. 5. Infrared thermographic derived $\mathrm{q}_{\text {surf }}$ for Shot 155648 at $3.5 \mathrm{~s}$ for the toroidal region $\phi=$ $98^{\circ}-115^{\circ}$. There are limiter apexes at $\phi=101.25^{\circ}$ and $108.75^{\circ}$, i.e. at $\mathrm{t}= \pm 0.0664 \mathrm{~m}$. Individual tiles are indicated by black dashed lines and white numbers.

Fig. 6. Shot 155648 at $4 \mathrm{~s}$. Radial profiles of extracted $q_{\| \text {ir }}$ for 6 vertical slices at toroidal angles indicated (Fig. 5).

Fig. 7. Normalized $q_{\|}$profiles for 155648 at 3-4 s. The $q_{\| \text {ir }}$ profile is the averaged data of the 6 profiles in Fig. 6. The lower arc of the SP extends further out radially than the upper arc (Fig. 1); however, the upper arc measuring locations are closer to the IWL, being $\sim 1 \mathrm{~m}$ away in the $\|$-direction, $\mathrm{cf} \sim 3 \mathrm{~m}$ for the lower arc. 


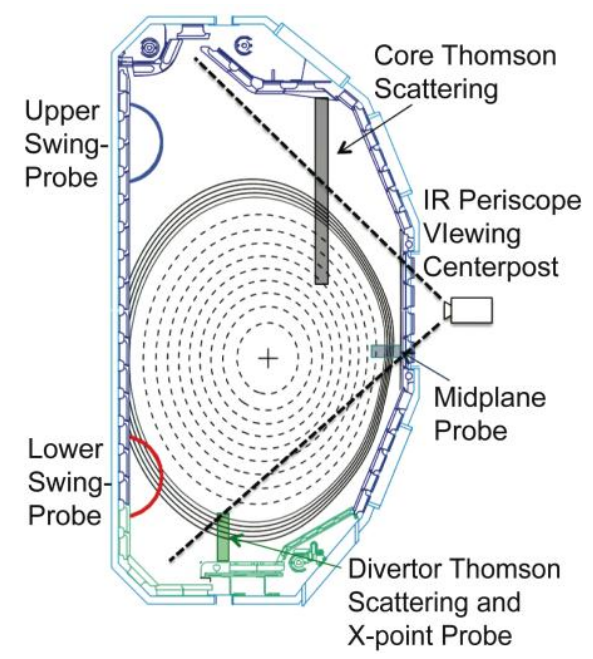

Fig. 1. $64 \mathrm{~mm}$

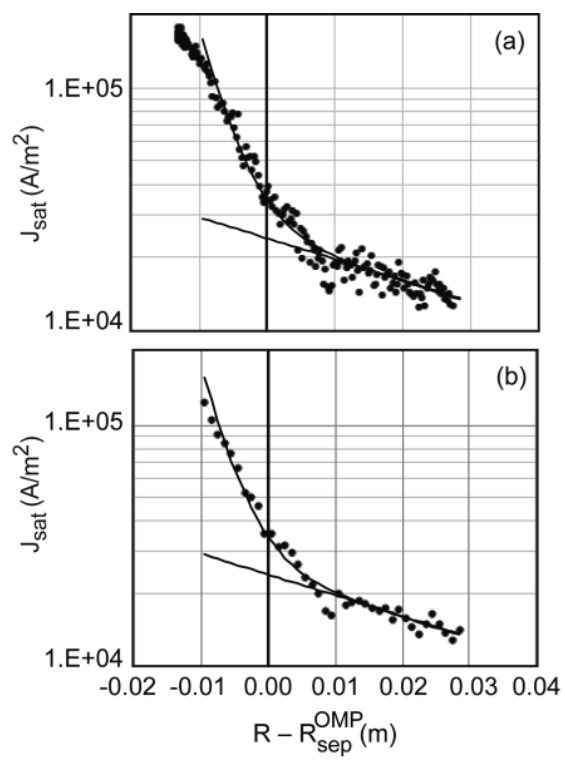

Fig. 2. $75 \mathrm{~mm}$ 


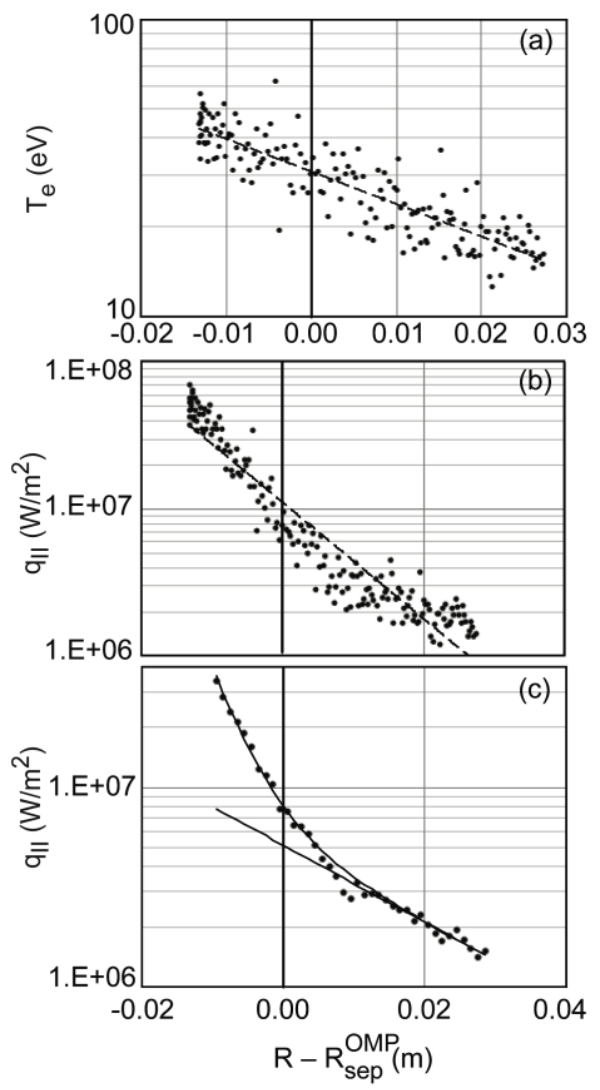

Fig 3. $110 \mathrm{~mm}$

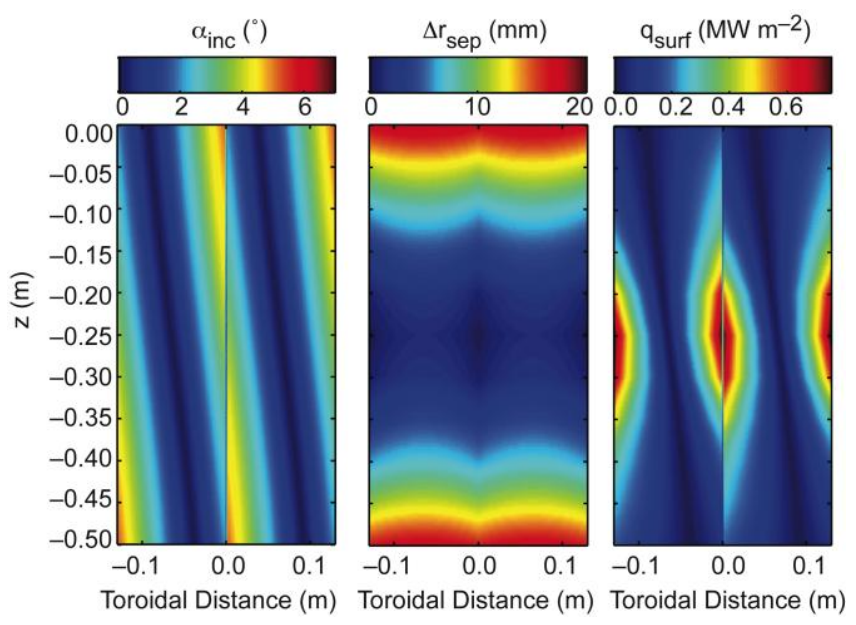

Fig. 4 (double column) $60 \mathrm{~mm}$ 


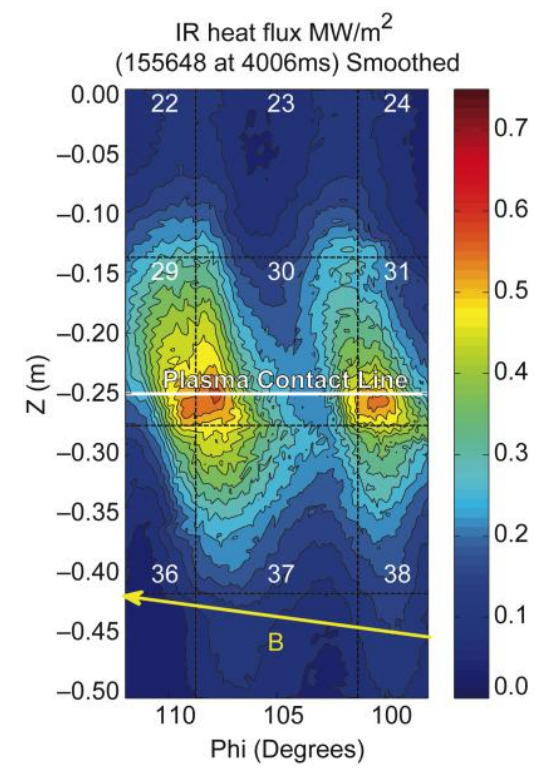

Fig. 5. $77 \mathrm{~mm}$

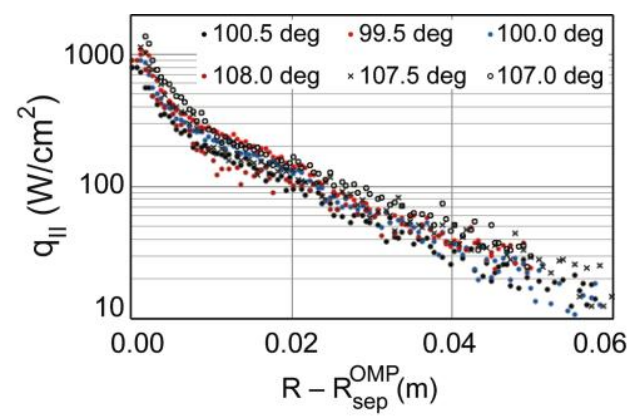

Fig. 6. $42 \mathrm{~mm}$

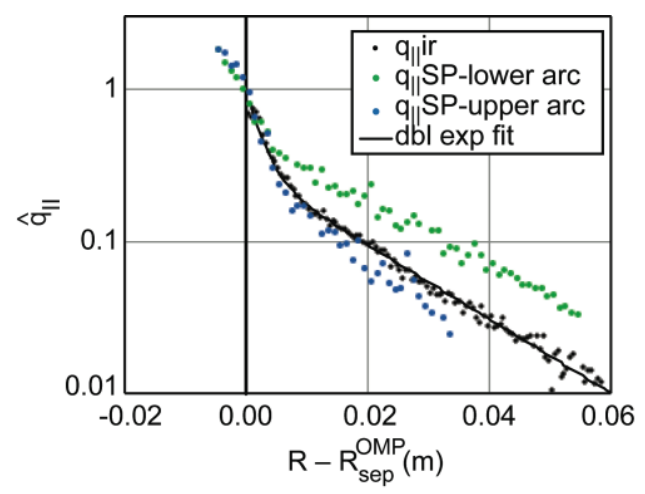

Fig. 7. $48 \mathrm{~mm}$ 